\title{
Fréquence et intensité des îlots de chaleur à rennes : bilan de 16 années d'observations (2004-2019)
}

\author{
V. Dubreuil *, X. Foissard, J. Nabucet, A. Thomas et H. Quénol \\ LETG Rennes, Université Rennes 2 / CNRS, UMR 6554, Rennes, France
}

\begin{abstract}
Résumé - Cet article s'intéresse à l'observation de l'îlot de chaleur urbain (ICU) à Rennes de 2004 à 2019. L'existence d'un réseau d'observation urbain relativement ancien et dense permet de quantifier la fréquence et l'intensité de l'ICU en fonction de l'heure et de la saison : l'ICU moyen atteint ainsi $1,3^{\circ} \mathrm{C}$ mais il approche $3^{\circ} \mathrm{C}$ en moyenne la nuit en fin d'été et peut parfois dépasser $7^{\circ} \mathrm{C}$. Les nuits de fort ICU, supérieur à $4^{\circ} \mathrm{C}$, représentent plus de $17 \%$ des observations en moyenne mais jusqu'à près de $30 \%$ en septembre. En 2019 comme en 2011, un nombre record d'ICU de forte intensité a été relevé, résultant essentiellement d'une forte fréquence de situations radiatives (forte insolation, vent faible). La spatialisation de l'ICU montre que le gradient classique centre-ville / périphérie n'est pas symétrique entre le sud et le nord de l'agglomération : la présence de parcs et de vallées au nord génère, en effet, des îlots de fraîcheur dont l'intensité varie en fonction de leur taille.
\end{abstract}

Mots-clés : îlot de chaleur urbain / Rennes / gel.

\begin{abstract}
Frequency and intensity of urban heat islands in Rennes: assessment of 16 years of monitoring (2004-2019). This article focuses on the observation of the urban heat island (UHI) in Rennes from 2004 to 2019. The existence of a relatively old and dense urban observation network makes it possible to quantify the frequency and intensity of the UHI according to the hour of the day and the season: the mean UHI thus reaches $1.3^{\circ} \mathrm{C}$ but it approaches $3^{\circ} \mathrm{C}$ on average at night in late summer and can sometimes exceed $7^{\circ} \mathrm{C}$. Strong UHI situations, above $4^{\circ} \mathrm{C}$, represent more than $17 \%$ of observations on average but up to almost $30 \%$ in September. In 2019 as in 2011, a record number of high intensity UHIs were noticed, essentially resulting from a high frequency of radiative situations (strong sunshine, weak wind). The spatialization of the UHI shows that the classic gradient between downtown and the periurban area is not symmetrical between the south and the north of the agglomeration: indeed, the presence of parks and valleys to the north generates islands of freshness whose intensity varies according to their size.
\end{abstract}

Keywords: Urban Heat Island / Rennes / Frost.

$\overline{\text { * Auteur de correspondance }}$ : vincent.dubreuil@univ-rennes2.fr

\section{Introduction}

L'observation du climat urbain a connu un essor considérable au cours des dernières décennies. On peut l'expliquer en partie par le fait qu'une part croissante (et majoritaire) de la population mondiale vit désormais en ville (ONU, 2015) : ainsi, les études sur les risques liés aux vagues de chaleur, de pollution et aux inondations ont nécessité la mise en place de dispositifs de mesures adaptés à l'échelle urbaine. La croissance rapide des villes des pays en développement a également entraîné une augmentation de l'exposition des populations et des activités urbaines, accroissant la vulnérabilité de ces territoires et expliquant l'intérêt croissant de l'étude des climats urbains dans ces pays (Oke et al., 2017 ; Amorim et Dubreuil, 2017). L'impact de l'urbanisation sur la formation de l'îlot de chaleur urbain a donné lieu à de nombreuses études dans les grandes métropoles comme Delhi (Mohan et al. 2009), Athènes (Kastoulis et al. 1985), New York (Gaffin et al. 2008) et Tokyo (Hung et al. 2006). Dans les plus grandes villes, l'îlot de chaleur urbain peut s'étendre au-delà de la périphérie sur plusieurs 
dizaines de kilomètres.

En France, pays anciennement et fortement urbanisé, les études sur le climat urbain ont longtemps été peu développées et limitées à l'agglomération parisienne (Dettwiller, 1970 ; Escourrou, 1991 ; Cantat, 2004). La préoccupation première pour la capitale a suivi le mouvement mondial de l'étude privilégiée des grandes agglomérations, celles dont l'impact sur le climat local est le plus intense. Il faut attendre le début des années 2000 pour voir émerger, plus tard qu'ailleurs, des études portant sur des villes de taille plus modeste. Il est probable que l'impact de la vague de chaleur de 2003 et la surmortalité urbaine qui en a résulté, puis la mise en place des plans climats à partir de 2006, ont été les éléments déclencheurs d'une série d'initiatives et de projets de recherches concernant la plupart des métropoles françaises comme à Marseille (Mestayer et al., 2005), Toulouse (Pigeon et al., 2007), Rennes (Dubreuil et al., 2008), Strasbourg (Kastendeutch et al., 2010), Nice (Carrega, 2013), Dijon (Richard et $a l ., 2018$ ) ou Lyon (Renard et al., 2019). Il en a résulté également une préoccupation plus importante portée d'emblée sur l'étude des îlots de chaleur urbain (ICU).

L'ICU est, en effet, le phénomène climatique le plus étudié et le plus emblématique de la modification du climat local par l'urbanisation. Comme il s'agit d'un phénomène marqué essentiellement la nuit, il a souvent été étudié à partir de campagnes de mesures itinérantes car le réseau conventionnel n'a pas une densité suffisante pour pouvoir l'appréhender (Masson et al., 2019). Mais pour observer cet ICU dans la durée, il faut disposer de réseaux de mesures à échelle fine. Pour ce faire, plusieurs équipes de recherches ont ainsi implanté depuis quelques années des réseaux de mesures permanents dédiés à ce phénomène mais, en France, contrairement à d'autres pays (Alcoforado et al., 2014), ce sont des réseaux récents. La situation est un peu différente à Rennes où un tel réseau existe depuis 2004 (Dubreuil et al., 2010).

A partir des observations faites par le réseau de suivi de l'ICU à Rennes, cet article propose donc de faire le bilan des observations réalisées sur 16 années complètes. La période d'observation est suffisamment longue pour analyser des indicateurs comme les fréquences d'intensités de l'ICU, définies comme la différence de température entre la station de référence rurale et celle du centre-ville, le nombre de jours de gel ainsi que la cartographie de quelques situations particulièrement représentatives en été et en hiver. Il s'agit aussi de revenir sur la particularité de l'année 2019, durant laquelle le record absolu de température a été atteint avec $40,1^{\circ} \mathrm{C}$ et avec une forte fréquence et intensité des ICU.

\section{Données et méthodes}

Pour étudier le phénomène d'ICU, un réseau de stations automatiques a été implanté à partir de 2004 dans le cadre du programme ECORURB (Dubreuil et al., 2010) : ce programme résultait d'une demande sociale forte en liaison avec la gestion délicate d'espèces aviaires invasives, ainsi que d'une volonté politique de mettre en place des actions visant à limiter le réchauffement climatique à la fois par des mesures d'atténuation des émissions de gaz à effet de serre (première ligne de métro ouverte en 2002) mais aussi de contribuer localement à tenter d'envisager des solutions pour réduire le phénomène d'îlot de chaleur urbain (Poquet et al., 2019).

En 2019, le réseau d'observation climatologique permanent de l'ICU à Rennes était constitué de 25 stations automatiques de type DAVIS Vantage Pro et Weather Monitor, auxquelles il faut ajouter les deux stations officielles gérées par Météo France et par l'INRA (figure 1). Sur la période d'étude, 20 stations sont réellement disponibles, certaines ayant été installées plus récemment (Vezin en 2008, écomusée en 2010). Certaines ont aussi dû être déplacées ou désactivées en raison de travaux sur sites et deux ont été vandalisées. Ce réseau principal offre une disponibilité totale des données d'environ $80 \%$ (figure 2) : les lacunes principales sont dues au remplacement progressif à partir de 2008 de la première génération de stations (Weather Monitor) par des modèles plus récents dont la mémoire n'est pas vidée lors d'une panne d'alimentation électrique. A partir de 2016 et l'automatisation de l'accès aux données par internet, les lacunes 
deviennent nettement moins importantes. La maintenance d'un tel réseau reste complexe et coûteuse en termes de ressources humaines, les discontinuités des disponibilités de personnels dédiés s'étant avérées particulièrement problématiques en 2008, 2013 et 2014.

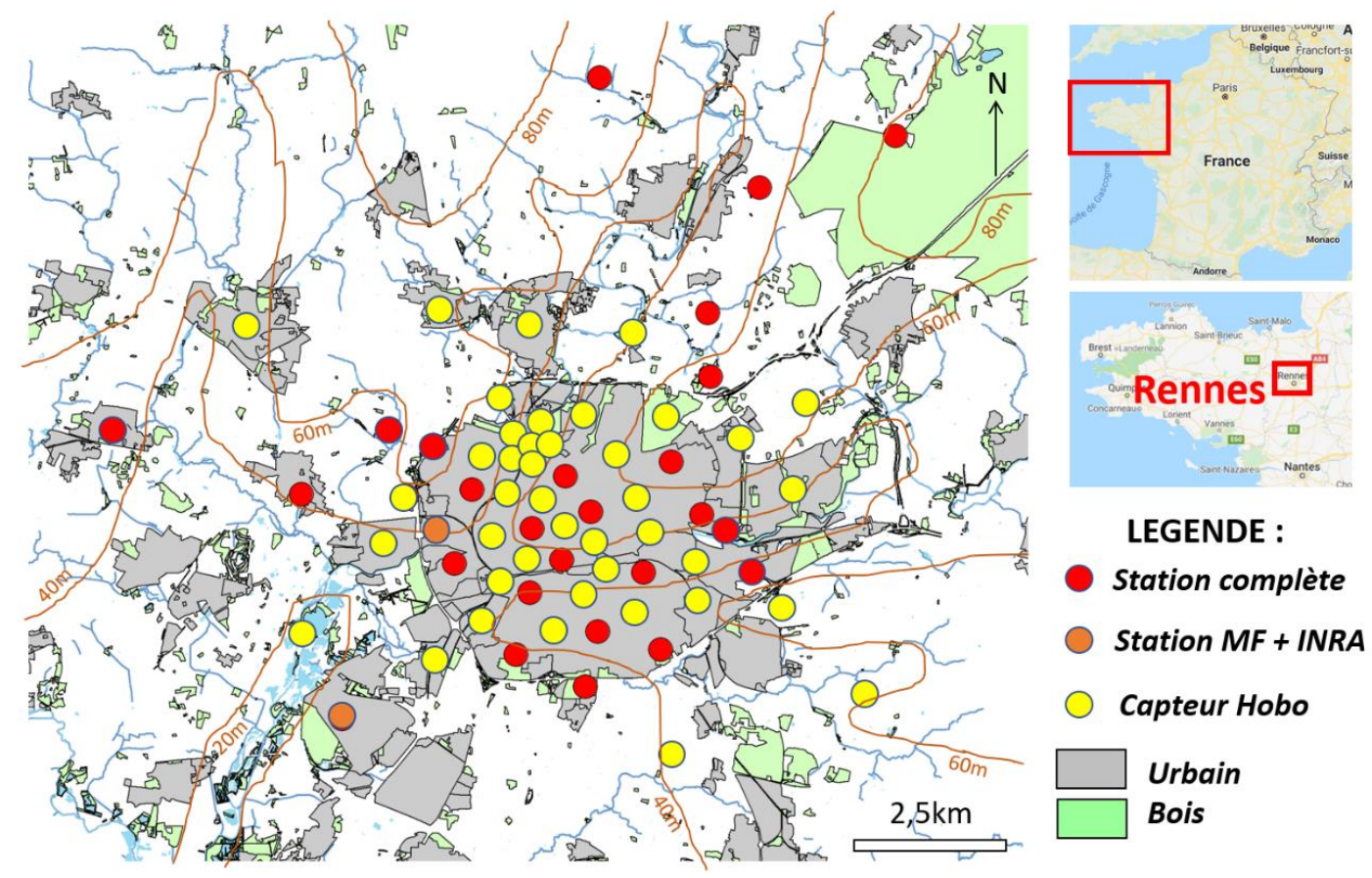

Figure 1. Réseau de mesure météorologique à Rennes en 2019 : en rouge, les stations complètes du réseau permanent ; en orange, les stations officielles de Météo France et de l'INRA. En jaune, réseau de sondes thermohygrométriques (campagnes saisonnières de 2011, 2016 et 2019). Courbes de niveau équidistantes de $20 \mathrm{~m}$. Meteorological network in Rennes in 2019: in red, the complete stations of the permanent network; in orange, the official stations of Météo France and INRA. In yellow, network of thermo-hygrometric sensors (work fields of 2011, 2016 and 2019). Equidistant 20 m elevation contour lines.

A ce réseau principal s'ajoute de manière ponctuelle un réseau de mesure de 25 capteurs HOBO et TINYTAG disposés sur l'ensemble de l'agglomération lors de campagnes hivernales et estivales réalisées pendant les années 2011, 2016 et 2019. Contrairement aux stations principales qui nécessitent une implantation en lieu sûr et permanent, ce réseau complémentaire plus léger peut être installé sur du mobilier urbain à une hauteur typique de trois mètres pour éviter vol et dégradation. Sur ce point, signalons que sur toute la période, deux stations et deux abris-légers ont subi des dégradations majeures rendant impossible l'utilisation des données.

Les critères de choix d'implantation des stations principales sont décrits dans des travaux antérieurs (Dubreuil et al., 2010 ; Foissard et al., 2019). La carte de la figure 3 permet de situer les points de mesure par rapport à leur environnement, défini ici par les LCZ (Local Climate Zone). Les LCZ ont été définies à partir des seuils de fractions d'occupation du sol (bâtie, végétation...), de l'effet de canyon (" sky view factor ») et de la hauteur moyenne des bâtiments dont les principaux descripteurs sont indiqués ci-après. Ces seuils correspondent aux propriétés des morphologies urbaines décrites par Stewart et Oke (2012).

Les stations peuvent être classées en trois grandes catégories :

1. Les stations rurales ou périurbaines situées à plus de 5 kilomètres du centre et dont la fraction de végétation dans un rayon de 500 mètres dépasse $55 \%$ et situées en contexte agricole ou rural correspondant aux LCZ 9 (sparsely built), B (scattered trees) ou D (low plants) : Melesse, Savio, La Lice, Morinais, Saint-Jacques, Ecomusée. 
Figure 2. Pourcentage de données manquantes (en rouge) du réseau d'observation climatique à Rennes de 2004 à 2019. Percentage of missing data (in red) from the climate observation network in Rennes from 2004 to 2019.

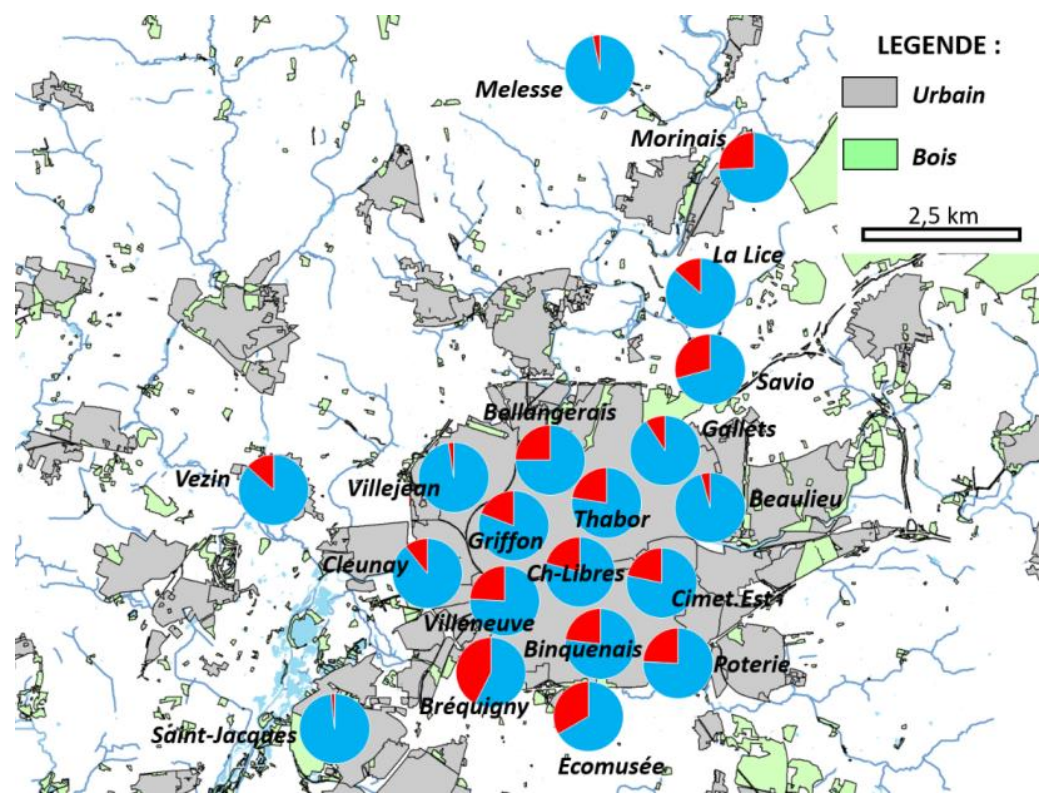

2. Les stations suburbaines à fort taux de surface imperméabilisée (plus de $30 \%$ ) ou construite (plus de $10 \%$ ), toutes (sauf une) à l'intérieur de la rocade et à moins de 4 kilomètres du centreville. Elles se répartissent entre les LCZ 5 (open mid-rise) des grands ensembles et petits collectifs (Bréquigny, Villejean, Cleunay, Villeneuve) avec une hauteur moyenne des bâtiments comprise entre 10 et $15 \mathrm{~m}$ et les quartiers pavillonnaires de LCZ 6 (Poterie, Cimetière de l'Est, Vezin, Gallets, Beaulieu) dont la hauteur moyenne des bâtiments est inférieure à $10 \mathrm{~m}$. La station du Thabor, située dans un parc urbain à proximité du centre peut être rattachée à ce groupe.

3. Les stations du centre-ville, ici classées en LCZ 2 (compact mid-rise) où la fraction de végétation est inférieure à $10 \%$, la surface en bâti supérieure à $35 \%$ et imperméabilisée comprise entre 50 et $60 \%$ : Griffon et ChampsLibres. La hauteur moyenne des bâtiments est supérieure à $10 \mathrm{~m}$ (l'ensemble des quartiers classés en LCZ 2 ont une hauteur moyenne de $14,2 \mathrm{~m})$.

Figure 3. Cartographie des Local Climate Zone à Rennes (LCZ) et localisation des stations météorologiques de suivi de l'ICU, situation en 2016. NB : les stations de la Morinais et Melesse sont horschamp et situées en LCZ D. Mapping of the Local Climate Zone in Rennes (LCZ) and location of the UHI meteorological monitoring stations, situation in 2016. NB: the Morinais and Melesse stations are out of the map and located in LCZ D.

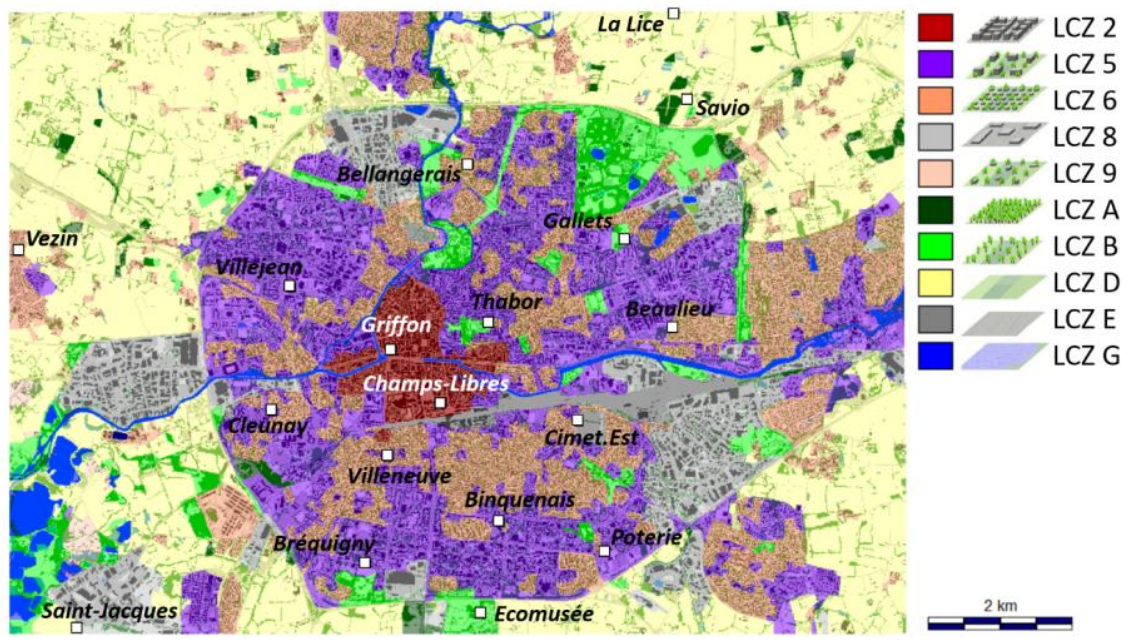

Avant d'étudier plus avant les données issues de ce réseau, il convient de vérifier que les mesures étalées sur 16 années n'ont pas connu de dérives majeures liées à des défauts de capteurs, au remplacement de ceux-ci au cours du temps ou même au changement de l'environnement lié à des 
travaux. La figure 4 présente ainsi les tests (purement visuels ici) réalisés pour contrôler les mesures effectuées dans 6 stations de ce réseau : elles appartiennent aux trois groupes définis plus haut et permettent de bien couvrir l'agglomération entre la partie rurale et périurbaine (Melesse et La Lice), suburbaine (Gallets, Poterie et Villejean) et centrale (Griffon). En raison des lacunes présentes certains mois, les données des stations voisines ont été utilisées ponctuellement pour obtenir des séries les plus complètes possibles ; certaines lacunes de 2007-2008 ou 2013-2014 n'ont cependant pas pu être comblées, comme expliqué plus haut. Pour chaque station, nous avons choisi de représenter chaque mois l'écart entre la moyenne des minimales (et des maximales) avec la station de référence, celle de Météo France à Saint-Jacques de la Lande.

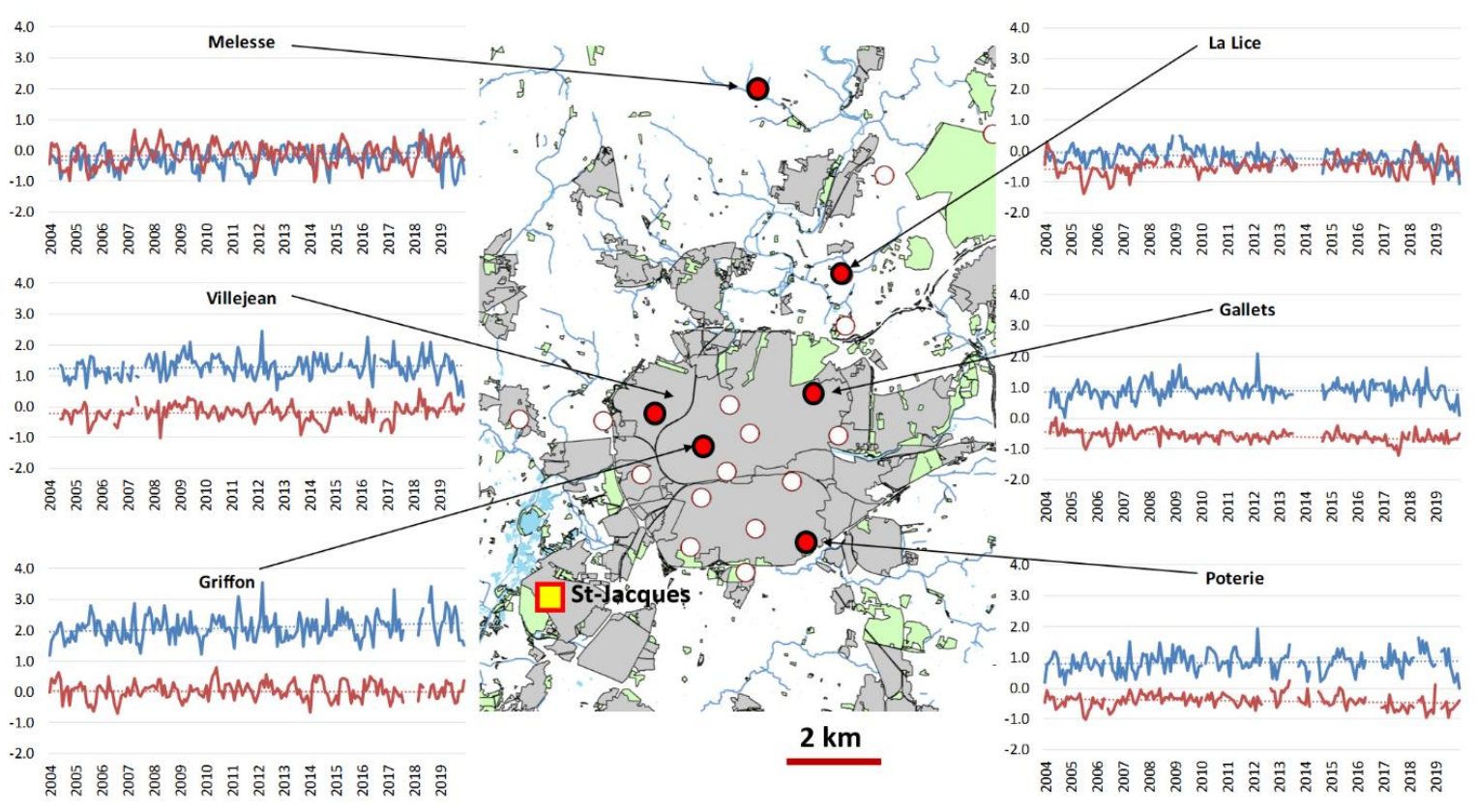

Figure 4. Ecart entre les moyennes minimales (en bleu) et maximales (en rouge) mensuelles avec Rennes-SaintJacques pour 6 stations de 2004 à 2019. Difference between the minimum (in blue) and maximum (in red) monthly averages with Rennes-Saint-Jacques for 6 stations from 2004 to 2019.

Les deux stations rurales (Melesse et la Lice) montrent des données en moyenne légèrement inférieures à celles de Saint-Jacques, surtout pour les minimales à Melesse $\left(-0,3^{\circ} \mathrm{C}\right)$ et pour les maximales à La Lice $\left(-0,5^{\circ} \mathrm{C}\right)$. L'écart avec SaintJacques reste stable au cours du temps sauf pour la Lice où on observe une légère tendance à la réduction des écarts entre minimales et maximales. Les stations suburbaines montrent des écarts plus significatifs pour les minimales : il atteint $0,8^{\circ} \mathrm{C}$ à la Poterie, $0,9^{\circ} \mathrm{C}$ aux Gallets et $1,3^{\circ} \mathrm{C}$ à Villejean, montrant que ces stations sont bien situées à l'intérieur de l'ICU rennais. L'écart est plus modeste pour les maximales, l'ensemble des stations étant plus fraîches que Saint-Jacques de quelques dixièmes de degrés, phénomène que l'on peut attribuer à la différence de capteur et d'abri utilisé ainsi qu'à l'altitude légèrement inférieure de la station de Saint-Jacques (35 mètres contre 65 mètres d'altitude pour les Gallets par exemple). Pour les Gallets et la Poterie, une très légère tendance à l'augmentation de l'écart avec SaintJacques (d'un à deux dixièmes) peut être noté mais aucune tendance n'apparaît sur les minimales. Enfin, la station du centre-ville de Griffon (complétée par celle des Champs Libres pour les mois manquants) est celle qui présente l'écart des minimales le plus important avec Saint-Jacques, soit $2^{\circ} \mathrm{C}$. C'est la seule qui présente une très légère tendance à la hausse de l'écart des minimales avec la station de référence: on pourrait y voir un accroissement au cours du temps de l'intensité de l'ICU au centre de Rennes mais c'est plus vraisemblablement l'effet des derniers mois de la série, ceux de 2019, qui ont connu des situations particulièrement favorables à celui-ci, nous $\mathrm{y}$ 
reviendrons. L'ensemble de ces données permet d'analyser maintenant le comportement temporel de l'ICU à Rennes avant d'aborder, dans un second temps, sa variabilité spatiale.

\section{Fréquences et intensités horaire et saisonnière de I'ICU à Rennes}

\subsection{Variations moyennes horaires et saisonnières de l'ICU à Rennes}

Pour cette étude, les données des températures minimales de deux stations de référence du centreville (Griffon et Champs Libres) et de deux stations de référence rurale (Melesse et Morinais au nord) sont analysées sur l'ensemble de la période (de 2004 à 2019), soit 4807 journées représentant $81 \%$ des journées possibles. Les deux couples de stations retenues, dont les données et les caractéristiques paysagères environnantes sont très proches, ne présentent de lacunes significatives qu'en 2004, 2008 et 2014, les données de la deuxième station de chaque binôme servant donc à combler les lacunes de la première. La base de données représente donc 16 années de mesures de l'intensité de l'ICU à pas de temps horaire qui permet d'établir les fréquences et intensités horaires et saisonnières de l'ICU mais aussi sa variabilité interannuelle. Les données de la station Météo France de Rennes Saint-Jacques sont également utilisées pour préciser le contexte climatique de la période étudiée, proche des normales trentenaires (1981-2010) avec $0,2^{\circ} \mathrm{C}$ d'écart avec la température moyenne, $0,6^{\circ} \mathrm{C}$ pour la température maximale et $-0,1^{\circ} \mathrm{C}$ pour la température minimale.

Tableau 1. Moyennes mensuelles des relevés à Rennes Saint-Jacques, Melesse (rural) et Griffon (centre-ville) de 2004 à 2019. Sigles: Tm, température moyenne ; Tn, température minimale ; Tx, température maximale ; Hum, humidité relative ; Pmm, précipitations ; Insol, insolation (en heures) ; Ff, vitesse moyenne du vent (m/s). Monthly averages in Rennes Saint-Jacques, Melesse (rural) and Griffon (downtown) from 2004 to 2019. Acronyms: Tm, average temperature; Tn, minimum temperature; Tx, maximum temperature; Hum, relative humidity; Pmm, precipitation; Insol, sunshine (in hours); Ff, mean wind speed ( $\mathrm{m} / \mathrm{s}$ ).

\begin{tabular}{|c|c|c|c|c|c|c|c|c|c|c|c|c|c|c|c|c|c|c|c|}
\hline & \multicolumn{7}{|c|}{ Moyennes Saint-Jacques 2004-2019 } & \multicolumn{6}{|c|}{ Moyennes Melesse 2004-2019 } & \multicolumn{6}{|c|}{ Moyennes Griffon 2004-2019 } \\
\hline & $\mathrm{Tm}$ & Tn & $\mathbf{T x}$ & Hum & Pmm & Insol & Ff & $\mathrm{Tm}$ & $\mathrm{Tn}$ & $\mathbf{T x}$ & Hum & Pmm & Ff & $\mathrm{Tm}$ & Tn & $\mathbf{T x}$ & Hum & Pmm & Ff \\
\hline J & & & & & 5.8 & & & & & 8.8 & 88.8 & & & 6.8 & 4.7 & 8.9 & 82.0 & 4. & 2.8 \\
\hline & & & & & & & & & & 10.0 & & & & & & 10.1 & & & 2.9 \\
\hline M & & & & & & & & & & & & & & & 9 & 3.1 & 1.6 & & 2.8 \\
\hline A & & & & & & & & 11.1 & 5.4 & 16.8 & 80.8 & 4.0 & 8 & 2.4 & 8.1 & 0.8 & 7.4 & & 2.8 \\
\hline M & & & & & & & & & & & & & & & & & & & \\
\hline J & .5 & .0 & & & & & 3 & 17.3 & 11.8 & 22.9 & 7 & 46.7 & 3.7 & & .9 & 3.0 & .2 & & 2.6 \\
\hline J & & & & & & & & 19.1 & 13.2 & 25.0 & .7 & .3 & 3.3 & & & 1.8 & 6 & & 2.7 \\
\hline A & & & & & & & & & & & & & & & & & & & 2.6 \\
\hline$S$ & 6.6 & 11.1 & 22.2 & 73.8 & 43.3 & 190 & 3.0 & 16.3 & 10.5 & 22.1 & 83.7 & 37.7 & 3.2 & 17.8 & 13.4 & 2.2 & 8.2 & & 2.7 \\
\hline 0 & & & & & & & & 13.1 & 8.8 & 17.4 & 89.3 & 69.8 & 3.5 & & 11.1 & 17.5 & 5.7 & +1.4 & 2.9 \\
\hline $\mathbf{N}$ & & & 12.6 & & & & & & & 12.3 & 90.6 & 74.7 & 3.6 & & 7.9 & 12.5 & 1.4 & & 2.9 \\
\hline D & 6.4 & 3.3 & 9.6 & 86.4 & \begin{tabular}{|l|}
68.6 \\
\end{tabular} & 73 & 3.5 & 6.2 & 3.3 & 9.1 & 89.7 & \begin{tabular}{|r|}
69.1 \\
\end{tabular} & 4.4 & 7.3 & 5.1 & 9.4 & 84.1 & 65.1 & 3.0 \\
\hline Moy. & 12.3 & 7.7 & 16.9 & 76.9 & \begin{tabular}{|l|}
671.0 \\
\end{tabular} & 1817 & 3.4 & 12.1 & 7.4 & 16.7 & 84.6 & \begin{tabular}{|c|}
670.1 \\
\end{tabular} & 3.8 & 13.2 & 9.7 & 16.8 & 73.6 & 567.6 & 2.8 \\
\hline
\end{tabular}

Les synthèses sur la période (tableau 1) permettent de rappeler le contexte climatique rennais, typique des régions tempérées océaniques de l'intérieur: températures et précipitations montrant un faible contraste saisonnier, une grande douceur l'hiver mais une chaleur peu marquée en été, saison présentant tout de même une baisse sensible des précipitations (Pmm), de la vitesse du vent (Ff) et de l'humidité relative (Hum). Selon la classification de Köppen-Geiger (Rubel et Kottek, 2010), le type moyen est de type Cfb (tempéré sans hiver froid ni été chaud et sans saison sèche) mais de nombreuses études (Eveno et al., 2016 ; Dubreuil et al., 2018) ont montré la récurrence des étés secs et la plus forte fréquence des types annuels Csb (tempéré à été frais et $\mathrm{sec}$ ).

Les valeurs moyennes montrent d'emblée la différence ville-campagne, surtout sur les températures minimales (Tn) avec plus de 2 degrés de différences entre Griffon et Melesse et SaintJacques. La ville apparaît également un peu plus sèche, moins arrosée et moins ventée que son 
alentour rural. La différence est insignifiante sur les maximales (Tx), rappelant que l'effet urbain est essentiellement un phénomène nocturne.

Ce phénomène peut être détaillé à partir de la figure 5 qui synthétise les moyennes horaires et mensuelles de l'intensité de l'ICU, pris ici comme la différence entre la station urbaine (Griffon) et rurale (Melesse). Des études antérieures (Dubreuil et al., 2010 ; Foissard et al., 2019) avaient déjà montré l'intérêt de ce type de représentation pour illustrer la temporalité de l'ICU mais, à chaque fois, uniquement sur des valeurs calculées sur une seule année. L'intérêt de la figure 5 est de moyenner cette approche sur 16 ans, ce qui permet de lisser l'effet de la variabilité interannuelle. Le cycle journalier de l'ICU apparaît clairement, quel que soit le mois : la différence ville-campagne est faible voire nulle le jour, surtout entre $10 / 11$ h et $15 / 16$ h $\left(0,2\right.$ à $0,3^{\circ} \mathrm{C}$ en moyenne) ; elle devient plus marquée en début de journée vers 7-8 h et en fin de journée vers 18-20 h (entre 1 et $1,5^{\circ} \mathrm{C}$ ) et devient forte la nuit avec une certaine stabilité de l'intensité de l'ICU (moyenne de 2 à $2,1^{\circ} \mathrm{C}$ ). On rappelle ici que les deux phénomènes majeurs expliquant cette différence sont: 1) la restitution de la chaleur accumulée pendant la journée par les bâtiments qui s'effectue en ville dès le coucher du soleil et jusqu'au jour suivant; 2) la présence plus importante de végétation dans la zone rurale qui, via l'évapotranspiration, dissipe l'énergie disponible sous forme de chaleur latente, diminuant en retour le flux de chaleur sensible responsable du réchauffement des basses couches de l'atmosphère (Oke, 1987 ; Arnfield, 2003 ; Masson, 2006 ; Lemonsu et al., 2012 ; Oke et al., 2017).

\begin{tabular}{|c|c|c|c|c|c|c|c|c|c|c|c|c|c|c|c|c|c|c|c|c|c|c|c|c|c|}
\hline ICU & $: 00$ & 01:00 & 02:00 & $03: 00$ & 04:00 & |05:00| & |06:00| & 07:00 & 08:00 & |09:00 & $10: 00$ & 11:00 & $12: 00$ & $13: 00$ & $\mid 14: 00$ & 15:00 & $\mid 16: 00$ & $17: 00$ & 18:00| & 19:00 & $20: 00$ & 21:00 & & & \\
\hline & & & & & & & & .3 & & & & & & 4 & & & & & & & & & & & \\
\hline & & & & & & & & & & & & & & & & & & & & & & & & & \\
\hline & & & & & & & & & & & & & & & & & & & & & & & & & \\
\hline & & & & & & 2 & 2.1 & & & & & & 0.0 & \begin{tabular}{|c|}
-0.1 \\
\end{tabular} & \begin{tabular}{|l|}
-0.1 \\
\end{tabular} & & & & & & 0 & & & & \\
\hline & & & & & & & & & & & & & & & & & & & & & & & & & \\
\hline & & & & & & & & & & & & & & & & & & & & & & & & & \\
\hline & & 2.3 & & & & 2.0 & 1.5 & 0.9 & 0.4 & & & 1 & & 0.2 & & & .2 & & 0.9 & 4 & .8 & & & 4 & \\
\hline & & & & & & & & & & & & & & & & & & & & & & & & & \\
\hline & & 8 & & & & 2.6 & 2.4 & & & & & & & & & & & & & & 2.8 & & & 10 & 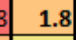 \\
\hline & & 1.9 & & & & 1.7 & 1.6 & 1.5 & & & & & & & & & & & & & .0 & & & & \\
\hline & & 1.6 & & 1.6 & 1.0 & 1.6 & 1.6 & .6 & 1.4 & & 0.7 & & 0.3 & & & & 0.0 & & & & 1.1 & & & & \\
\hline & & 1.4 & & 1.4 & 1 & 1.4 & 1. & 1.4 & & & & & & & & & & & & & & & & & \\
\hline & & 2.0 & & 2.0 & & 1.9 & & & 1.0 & & 0.4 & & & & & & & & & & .9 & & . & & \\
\hline
\end{tabular}

Figure 5. Variabilité horaire et saisonnière de l'ICU à Rennes de 2004 à 2019 : différence moyenne mensuelle par heure (colonne) et par mois (ligne) entre la température de la station de référence rurale (Melesse) et celle du centreville (Griffon) (heures T.U.). Hourly and seasonal variability of the UHI in Rennes from 2004 to 2019: average monthly difference per hour (column) and per month (row) between the temperature of the rural reference station (Melesse) and the city center (Griffon) (all in GMT).

La saisonnalité de l'ICU est également bien marquée sur la figure 5: le contraste thermique ville-campagne est plus marqué d'avril à septembre quand la nuit, la différence dépasse souvent en moyenne $2,5^{\circ} \mathrm{C}$. Les mois de fin d'été (août et septembre) sont ceux durant lesquels l'intensité moyenne de l'ICU est la plus forte: ils correspondent aux mois cumulant des valeurs moyennes de pluie et de vent plus faibles, des conditions qui favorisent le développement de l'ICU (Morris et Simmonds, 2001). La quantité d'énergie accumulée par la ville en fin d'été est maximale en septembre lorsque la restitution nocturne permet d'enregistrer des écarts moyens de près de $3^{\circ} \mathrm{C}$ entre le centre-ville et la campagne. Audelà de ces moyennes, il s'agit maintenant de préciser les intensités réelles des ICU observés à Rennes.

\subsection{Fréquences des intensités d'ICU à Rennes}

Nous avons choisi de classer ici les journées par gamme d'intensité de $\Delta \mathrm{Tn}$ de $2^{\circ} \mathrm{C}$ et plus (d'après Fernandez Garcia, 1996), en retenant la différence entre la minimale observée chaque jour à Melesse 
(rural) et Griffon (centre-ville) : il s'agit en quelque sorte d'une empreinte simple de l'ICU, même si tous ces écarts $\Delta \mathrm{Tn}$ ne correspondent pas systématiquement à l'ICU. Les journées durant lesquelles ce seuil est dépassé atteignent une fréquence de 50,6\% à Rennes, tous mois confondus et sur l'ensemble de la période, soit un peu plus d'un jour sur deux (figure 6). Au cours de l'année, les fréquences varient entre $64,6 \%$ en septembre, la fin de l'été étant plus favorable à l'accumulation de chaleur en ville, et 31,6 \% en janvier : en hiver, malgré la fourniture anthropique de chaleur (chauffage, trafic), l'absence de croissance végétale et de dissipation d'énergie sous forme de chaleur latente et le faible apport calorifique diurne l'emportent très largement pour expliquer la

Figure 6. Fréquences mensuelles des classes d'intensité d'ICU quotidien à Rennes de 2004 à 2019 : différences entre les minimales observées entre Griffon (centre) et Melesse (rural). Monthly frequencies of daily UHI intensity classes in Rennes from 2004 to 2019: differences between the minimum observed between Griffon (downtown) and Melesse (rural). réduction de l'ICU. Les journées lorsque l'ICU dépasse $4^{\circ} \mathrm{C}$ représentent $17,1 \%$ des cas (62 jours par an en moyenne) mais moins de $10 \%$ en décembre et janvier contre près de $30 \%$ en septembre. Les journées avec des ICU à plus de $6^{\circ} \mathrm{C}$ représentent $1,4 \%$ des observations (5 jours en moyenne par an): elles ne se sont produites pratiquement qu'entre mars et octobre et à de rares exceptions en mai : ce mois est, en effet, en moyenne légèrement plus humide et pluvieux qu'avril et surtout juin. En 16 ans, 8 journées au total ont dépassé le seuil de $7^{\circ} \mathrm{C}$, octobre et septembre regroupant 5 des 8 journées en question, et le record est détenu par l'ICU du $1^{\mathrm{er}}$ octobre 2011 avec $8^{\circ} \mathrm{C}$.

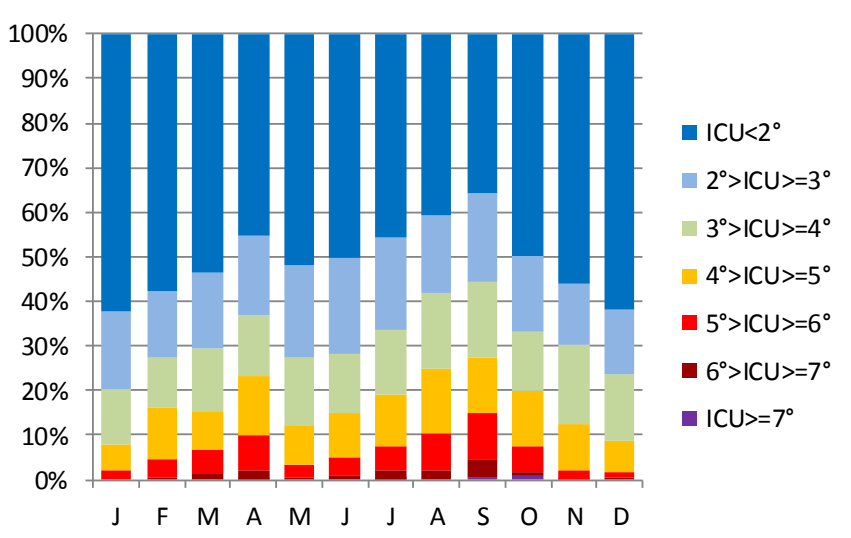

intensité (plus de $5^{\circ} \mathrm{C}$ ) a atteint 42 , soit autant qu'en 2011, précédent record ; on a même relevé 14 jours d'ICU supérieur à $6^{\circ} \mathrm{C}$, c'est-à-dire trois fois la valeur normale! Plus d'un jour sur trois (contre un sur quatre en moyenne) a enregistré un ICU supérieur à $3^{\circ} \mathrm{C}$, valeur record observée également en 2011 et les journées de faible ICU (moins de $2^{\circ} \mathrm{C}$ ) n'ont représenté que $42,5 \%$ du total, le record étant détenu par 2014 avec $41 \%$.

Afin d'interpréter ces différences, les données moyennes de Rennes Saint-Jacques pour chacune de ces années sont rappelées dans le tableau 2 ainsi que l'ICU moyen, maximum et la fréquence des ICU supérieurs à $4^{\circ} \mathrm{C}$, les plus significatifs (Foissard, 2015). Sur ces trois critères, 2011 et 2019 ressortent nettement. 2011 et 2014 étaient les deux années record en termes de températures moyennes $\left(12,9^{\circ} \mathrm{C}\right)$ et minimales $\left(8,3^{\circ} \mathrm{C}\right.$ pour les deux $): 2019$ a été légèrement moins chaude mais elle a surtout 
été très ensoleillée (plus fort ensoleillement observé de février à septembre depuis 1989). Il s'agit aussi d'une année contrastée sur le plan pluviométrique avec un début d'année peu arrosé (seulement 377 $\mathrm{mm}$ de pluie de janvier à septembre 2019) et de forts cumuls sur les trois derniers mois qui entraînent une valeur annuelle supérieure à la moyenne. Les situations radiatives, malgré un vent moyen record sur la période, ont donc prédominé en 2019 et permis le développement de situations de fort ICU en été comme en hiver. Ainsi, parmi les quatre mois avec l'ICU le plus marqué en 2019, on retrouve les mois d'été (juillet, août et septembre plutôt que juin) mais aussi le mois de février.
Figure 7. Fréquences annuelles des classes d'intensité d'ICU quotidien à Rennes de 2004 à 2019: différences entre les minimales observées entre Griffon (centre) et Melesse (rural). Annual frequencies of daily $\mathrm{UHI}$ intensity classes in Rennes from 2004 to 2019: differences between the minimum observed between Griffon (downtown) and Melesse (rural).

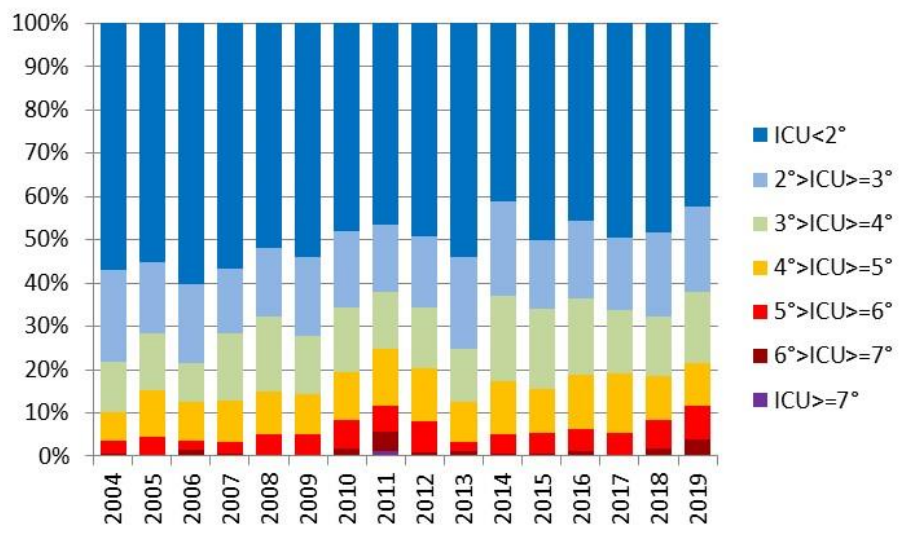

série sont aussi celles où les intensités d'ICU sont les plus faibles (avec 2013) mais ce sont des années dont les températures et les précipitations (sauf 2005) sont assez proches de la moyenne de la période. Elles participent cependant à l'impression d'une tendance à l'augmentation de l'intensité moyenne et de l'augmentation de la fréquence des journées de fort ICU perceptible sur la figure 7. ensoleillement qui ont permis une fréquence élevée des forts ICU. Les quatre premières années de la

Tableau 2. Intensités de l'ICU (haut) et moyennes annuelles à Rennes Saint-Jacques (en bas, RSJ) de 2004 à 2019. Les valeurs en bleu correspondent au premier quartile pour chaque série et celles en rouge au dernier quartile. Valeurs extrêmes en gras. Moy. ICU= moyenne annuelle de l'ICU quotidien (différence de Tn entre Griffon et Melesse) ; Max.ICU = écart maximal observé entre Griffon et Melesse. Fréq.ICU>=4 : pourcentage des jours où la différence de Tn entre Griffon et Melesse a été supérieure ou égale à $4^{\circ} \mathrm{C}$. Pour les autres sigles, se reporter au tableau 1 . UHI intensities (top) and annual averages in Rennes Saint-Jacques (bottom, RSJ) from 2004 to 2019. The values in blue correspond to the first quartile for each series and those in red to the last quartile. Extreme values in bold. Avg. ICU = annual average of daily UHI (difference in Tn between Griffon and Melesse); Max.ICU = maximum difference observed between Griffon and Melesse. ICU freq > = 4: percentage of days when the difference in Tn between Griffon and Melesse was greater than or equal to $4^{\circ} \mathrm{C}$. For other acronyms, see table 1.

\begin{tabular}{|l|r|r|r|r|r|r|r|r|r|r|r|r|r|r|r|r|}
\hline ICU Rennes & $\mathbf{2 0 0 4}$ & $\mathbf{2 0 0 5}$ & $\mathbf{2 0 0 6}$ & $\mathbf{2 0 0 7}$ & $\mathbf{2 0 0 8}$ & $\mathbf{2 0 0 9}$ & $\mathbf{2 0 1 0}$ & $\mathbf{2 0 1 1}$ & $\mathbf{2 0 1 2}$ & $\mathbf{2 0 1 3}$ & $\mathbf{2 0 1 4}$ & $\mathbf{2 0 1 5}$ & $\mathbf{2 0 1 6}$ & $\mathbf{2 0 1 7}$ & $\mathbf{2 0 1 8}$ & $\mathbf{2 0 1 9}$ \\
\hline Moy. ICU & 2.0 & 2.1 & 1.9 & 2.1 & 2.2 & 2.2 & 2.4 & 2.6 & 2.4 & 2.1 & 2.5 & 2.3 & 2.4 & 2.4 & 2.3 & 2.7 \\
\hline Max. ICU & 6.1 & 5.7 & 7.7 & 6.1 & 6.2 & 6.1 & 6.9 & 8.0 & 7.4 & 6.5 & 6.1 & 7.0 & 6.2 & 6.2 & 6.8 & 7.1 \\
\hline Fréq.ICU>=4 & 10.1 & 15.2 & 12.5 & 12.9 & 14.8 & 14.5 & 19.5 & $\mathbf{2 4 . 9}$ & 20.3 & 12.5 & 17.3 & 15.6 & 18.9 & 19.2 & 18.5 & 21.4 \\
\hline Moyennes RSJ : & & & & & & & & & & & & & & & & \\
\hline Tn (RSJ) & 7.7 & 7.6 & 8.2 & 7.8 & 7.4 & 7.2 & 6.4 & 8.3 & 7.4 & 7.4 & $\mathbf{8 . 3}$ & 7.9 & 7.5 & 7.9 & 8.1 & 8.0 \\
\hline Tx (RSJ) & 16.6 & 17.0 & 17.2 & 16.6 & 16.2 & 16.6 & 15.9 & 17.6 & 16.3 & 16.2 & 17.4 & 17.3 & 16.8 & 17.5 & 17.7 & 17.4 \\
\hline Tm (RSJ) & 12.2 & 12.3 & 12.7 & 12.2 & 11.8 & 11.9 & 11.1 & 12.9 & 11.9 & 11.8 & 12.9 & 12.6 & 12.1 & 12.7 & 12.9 & 12.7 \\
\hline Pmm (RSJ) & 621 & 532 & 653 & 722 & 759 & 731 & 549 & 516 & 785 & 747 & 856 & 596 & 625 & 635 & 659 & 748 \\
\hline Insol (RSJ) & 1742 & 1843 & 1750 & 1709 & 1760 & 1915 & 1969 & 1923 & 1731 & 1710 & 1835 & 1851 & 1680 & 1736 & 1951 & 1963 \\
\hline Ff (RSJ) & 3.5 & 3.5 & 3.6 & 3.6 & 3.5 & 3.4 & 3.5 & 3.4 & 3.4 & 3.5 & 3.4 & 3.5 & 3.3 & 3.2 & 3.5 & 3.6 \\
\hline Tx-Tn (RSJ) & 8.9 & 9.3 & 9.0 & 8.7 & 8.8 & 9.4 & 9.5 & 9.4 & 8.9 & 8.8 & 9.1 & 9.3 & 9.3 & 9.6 & 9.6 & 9.5 \\
\hline
\end{tabular}


On se gardera bien ici de tirer une significativité d'une tendance établie sur 16 années et on remarquera qu'une même tendance pourrait être observée concernant les températures (notamment l'amplitude Tx-Tn) et l'insolation (Insol dans le tableau 2, exprimée par la somme horaire annuelle). C'est donc plutôt l'augmentation de la fréquence des situations favorables au développement de l'ICU qui explique leur plus grande occurrence sur les dernières années plus qu'une amplification du phénomène lié à une croissance de l'agglomération rennaise. Ceci nous amène à la question de la distribution spatiale de l'ICU.

\section{Variabilité spatiale de l'ICU à Rennes}

L'approche spatiale va se focaliser sur l'année 2019, durant laquelle l'ensemble du réseau d'observation a pu être mobilisé pour établir des cartographies à échelle fine. On ne reviendra pas ici sur les méthodes d'interpolation spatiale utilisant des buffers de tailles variables en fonction des corrélations obtenues entre les classes d'occupation du sol et l'intensité de l'ICU pour chaque point de mesure. Les détails de la méthodologie sont décrits dans Amorim et al., 2015 et Foissard et al., 2019.

\subsection{Un ICU dont l'intensité et la configuration spatiale changent selon les mois}

La figure 8 permet de caractériser la configuration spatiale de l'ICU moyen en 2019, défini ici comme la différence entre la température moyenne minimale la plus basse enregistrée sur l'ensemble du réseau et chacune des stations. Cette intensité atteint $2,7^{\circ} \mathrm{C}$ en moyenne sur l'année, un gradient relativement fort puisqu'il s'exprime sur moins d'une dizaine de kilomètres entre le centreville et la campagne. Ce gradient est encore plus resserré au nord du centre historique en direction des prairies Saint-Martin où près de $2^{\circ} \mathrm{C}$ d'écart peuvent être observés : on cumule ici, en effet, un effet topographique (creux d'un ancien méandre de l'Ille), un effet de zone humide (secteur traditionnel d'épanchement des crues de la rivière) et un effet d'occupation des sols dans un secteur peu urbanisé et largement végétalisé (Nabucet, 2018) ; ce secteur apparaît fortement connecté sur le plan climatique avec les quartiers nord où la végétation est fortement présente dans des ensembles pavillonnaires assez aérés et de larges avenues pénétrantes et bordées d'arbres.

La configuration spatiale de l'urbanisme et de la topographie oppose ainsi la partie nord de la ville, avec un schéma assez peu concentrique, et la partie sud où le gradient climatique entre le centre et la périphérie suit un gradient beaucoup plus régulier. Une même dissymétrie peut être observée entre l'est et l'ouest : à l'ouest, le gradient thermique est assez bien marqué (à l'exception de la Zone Industrielle de la route de Lorient) alors qu'à l'est, la limite est plus diffuse dans les vastes secteurs pavillonnaires de la commune de Cesson-Sévigné. De manière générale, cette configuration se retrouve pour les quatre mois de 2019 représentés sur la figure 8 et paraît assez déconnectée des limites des LCZ de la figure 3, montrant le caractère diffus du réchauffement climatique urbain nécessitant la prise en compte de l'occupation des sols et des types de bâtis à échelle fine (Richard et al., 2018 ; Hidalgo et al., 2019). Dans le détail, des nuances saisonnières apparaissent cependant.

En hiver, même si, en moyenne, l'intensité de l'ICU est moins forte en saison froide, certains mois peuvent faire exception. Ce fut notamment le cas de février 2019 (figure 8), marqué par des conditions quasi-printanières : ce mois a été particulièrement remarquable en termes d'ensoleillement (record historique de 145 heures sur le mois), de températures maximales, deuxième valeur la plus élevée jamais enregistrée à Rennes, et par un vent moyen inférieur de près d'un mètre par seconde à la valeur moyenne. Les minimales moyennes dépassent ainsi $5^{\circ} \mathrm{C}$ dans le centre-ville contre $3^{\circ} \mathrm{C}$ à Saint-Jacques et un peu moins de $2^{\circ} \mathrm{C}$ dans les stations rurales du nord de l'agglomération, soit $3,6^{\circ} \mathrm{C}$ d'ICU moyen sur l'ensemble du mois, c'està-dire trois fois la valeur moyenne! Lors de la matinée du 12 février 2019 (figure 9), une des plus froides du mois, alors qu'il gelait à Saint-Jacques de la Lande et dans tout le bassin rennais, la température est restée largement positive en ville ; au cours des deux nuits suivantes, seules les stations les plus éloignées de la ville sont restées avec des températures négatives. 

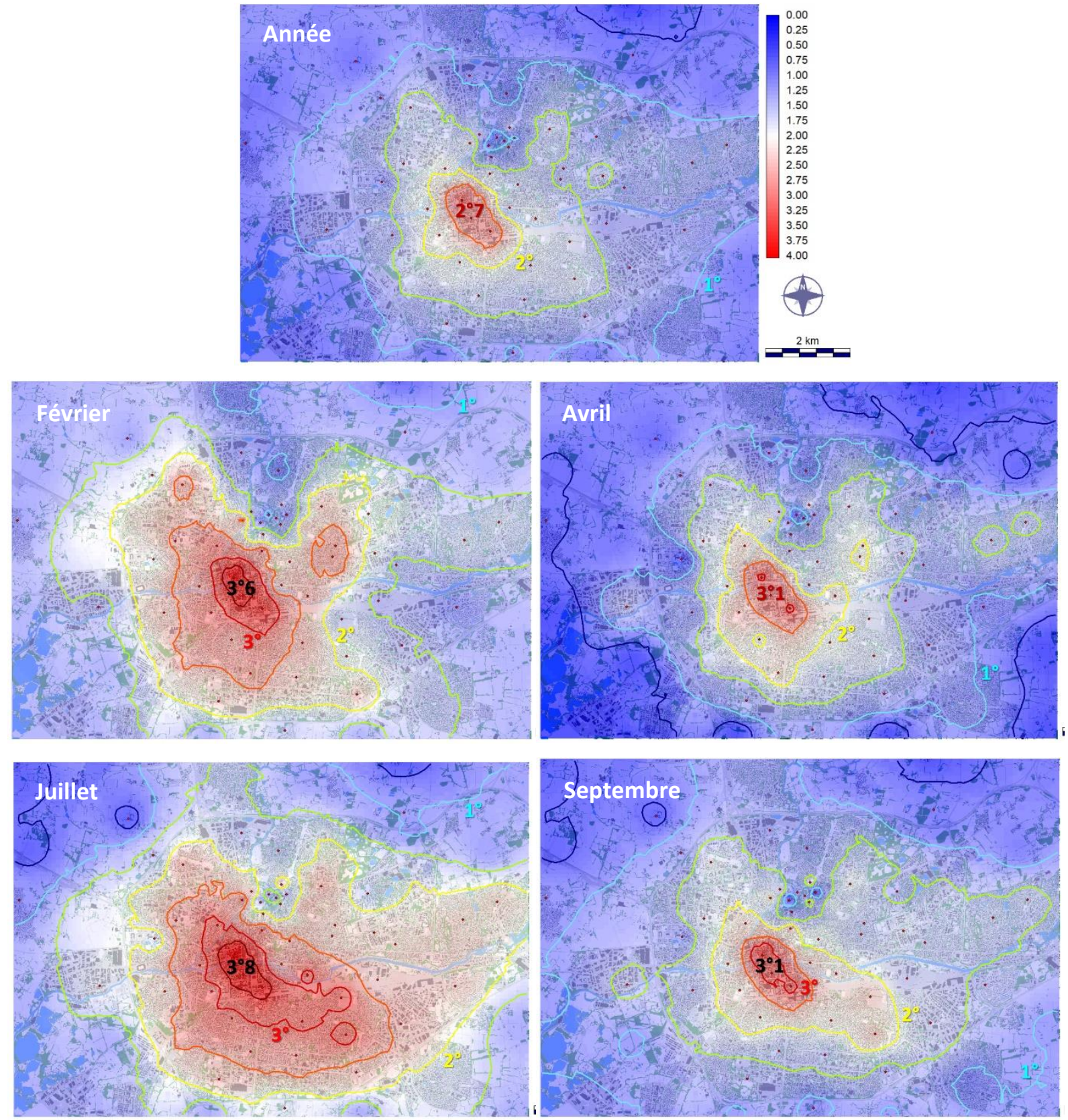

Figure 8. Intensité moyenne de l'îlot de chaleur à Rennes en 2019 (différence avec la température minimale moyenne de la station de la plus froide). En haut, valeur moyenne annuelle; au milieu à gauche, février, à droite, avril. En bas à gauche, juillet, à droite, septembre. Légende identique pour les 5 cartes et isothermes tous les $0,5^{\circ} \mathrm{C}$. Average intensity of the urban heat island in Rennes in 2019 (difference with the average minimum temperature of the coldest station). Above, annual average value; middle left, February, right, April. Bottom left, July, right, September. Identical legend for the 5 cards and isotherms every $0.5^{\circ} \mathrm{C}$.

Les mois d'avril et septembre (figure 8) présentent des intensités d'ICU comparables, d'un peu plus de $3{ }^{\circ} \mathrm{C}$ si on considère les minimales moyennes. La configuration spatiale montre cependant un ICU plus resserré en avril avec l'isotherme $1^{\circ} \mathrm{C}$ qui reste proche de la limite construite de la rocade (sauf à l'est). Ce sont deux mois plutôt plus ensoleillés et moins arrosés que la normale et fortement contrastés du point de vue des températures (alternance de périodes plus chaudes et plus froides que la normale). La principale différence entre ces deux mois vient du vent plus soutenu en septembre $\left(4 \mathrm{~m} . \mathrm{s}^{-1}\right.$ en moyenne à SaintJacques) qu'en avril $\left(3,3 \mathrm{~m} \cdot \mathrm{s}^{-1}\right)$. 


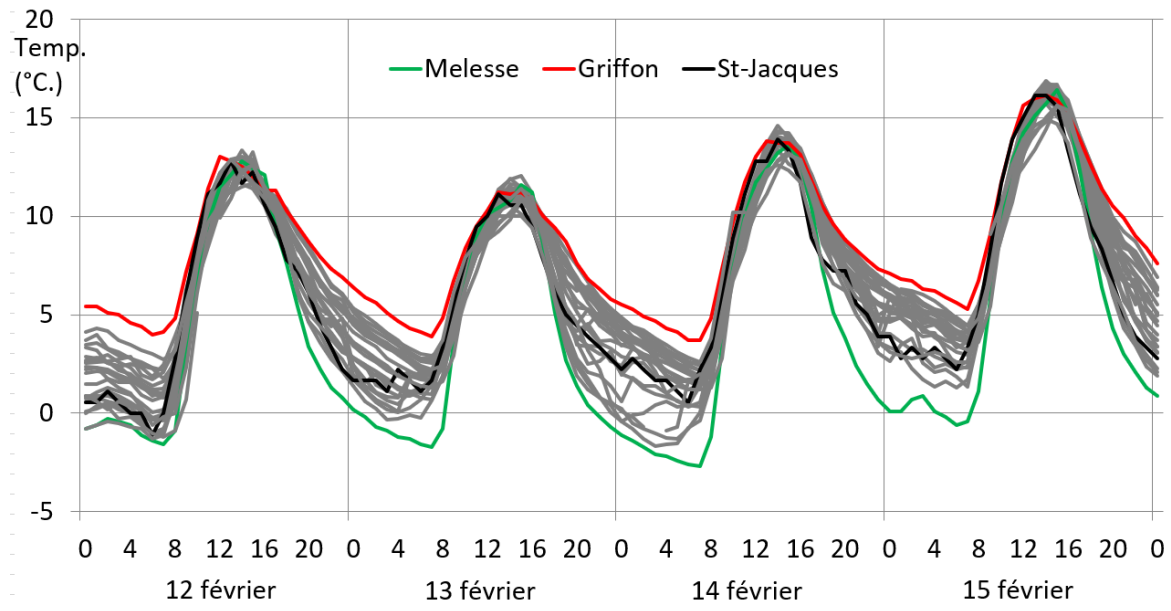

Figure 9. Variations horaires de la température du 12 au 15 février 2019 à Rennes (heures T.U. en abscisses). Seules les courbes de la campagne (Melesse en vert), du centre (Griffon en rouge) et de Saint-Jacques (en noir) sont différenciées, les courbes des autres stations sont en gris. Hourly temperature variations from February 12 to 15, 2019 in Rennes (GMT hours on the abscissa). Only the curves of the countryside (Melesse in green), the center (Griffon in red) and Saint-Jacques (in black) are differentiated, the curves of the other stations are in gray.

En été, la dernière carte de la figure 8 , le mois de juillet, permet de visualiser une situation de très fort ICU estival. Rappelons que l'été 2019 a connu deux vagues de chaleur importantes en France au cours des mois de juin et juillet. A cette occasion, de nombreux records de maxima absolus ont été battus un peu partout et Rennes n'y a pas fait exception : ainsi, le record absolu d'août 2003 de $39,5^{\circ} \mathrm{C}$ a été dépassé par la mesure du 23 juillet 2019 atteignant $40,1^{\circ} \mathrm{C}$ ! A la fin du mois de juin, un premier épisode caniculaire avait déjà fait monter les températures à Rennes jusqu'à $35,7^{\circ} \mathrm{C}$. Pour autant, les moyennes des minimales comme des maximales n'ont pas atteint des valeurs exceptionnelles : le mois le plus chaud a été juillet avec une moyenne de $21,1^{\circ} \mathrm{C}$ ce qui implique que, du point de vue de la classification de Köppen, l'année est restée de type Csb (c'est-à-dire le type le plus fréquent à Rennes) et pas Csa comme 2006 ou 2003 (Eveno et al., 2016 ; Dubreuil et al., 2018). Pourtant, en ville, la sensation de chaleur a souvent été plus forte, en raison du phénomène d'ICU, comme l'illustre la situation de juillet (figure 10). Lors de cet épisode caniculaire, la température n'est pas descendue en dessous de $20^{\circ} \mathrm{C}$ dans le centre-ville pendant 4 jours, du 23 au 26 juillet : la plus forte intensité de l'ICU a été relevée pendant la nuit du 24 au 25 avec une amplitude sur les minimales de près de $7^{\circ} \mathrm{C}$. La représentation spatiale montre l'accumulation de chaleur qui s'est produite dans le centre-ville mais aussi dans les quartiers du centre-sud où plusieurs capteurs ont enregistré de jour des températures absolues supérieures à $40^{\circ} \mathrm{C}$ et, de nuit, des minimales qui sont restées au-dessus de $24^{\circ} \mathrm{C}$ ! Comme sur les autres cartes, le secteur nord a conservé des températures plus basses à proximité du centre-ville (ICU souvent inférieur à $2^{\circ} \mathrm{C}$ ) alors que les quartiers sud jusqu'à la rocade sont restés dans cette enveloppe d'ICU supérieur à $2^{\circ} \mathrm{C}$. La question des îlots de fraîcheurs intra-urbains est ainsi posée.

Les cartes de la figure 8 montrent dans le détail comment l'ICU a été modulé par la présence de zones végétalisées, ce que de nombreux auteurs ont désigné comme des îlots de fraîcheur intra-urbains et décrits un peu partout dans le monde (Chang et al., 2007 ; Oliveira et al., 2011 ; Yang et al., 2017). C'est le cas de l'ensemble des espaces verts autour des prairies Saint-Martin (30 hectares au nord de la commune à proximité immédiate du centre-ville) : le gradient instantané observé y est souvent très important, supérieur à $4^{\circ} \mathrm{C}$, par exemple lors de la nuit du 24 au 25 juillet 2019. C'est aussi le cas pour le Parc du Thabor, également proche du centre historique, dont la température est restée 2 degrés inférieure à celle du centre-ville pendant cette séquence. 


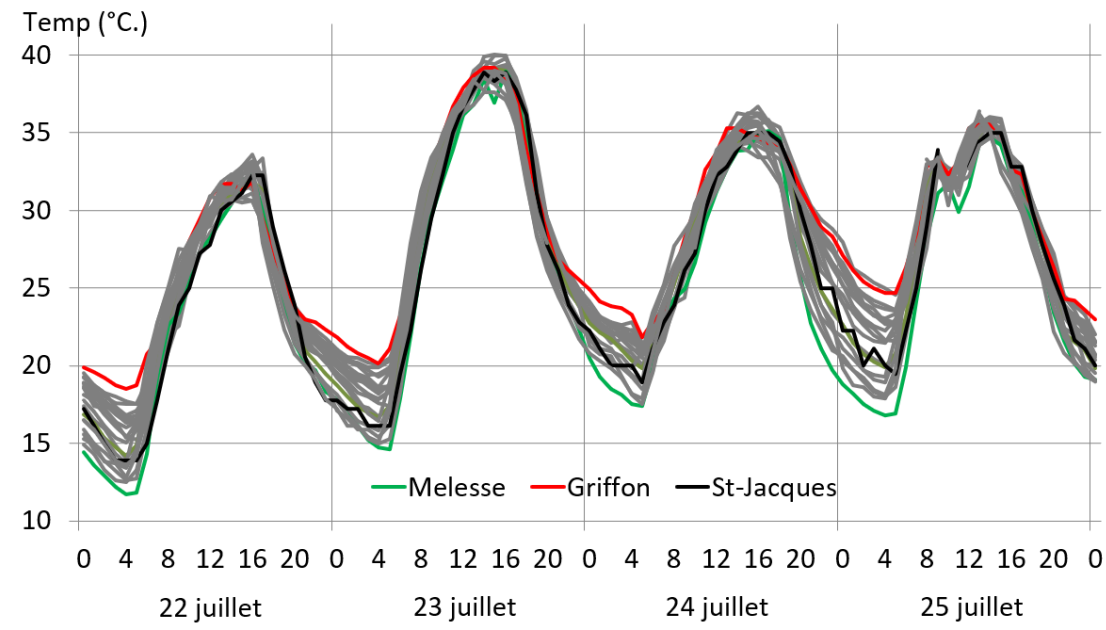

Figure 10. Variations horaires de la température du 22 au 25 juillet 2019 à Rennes (heures T.U. en abscisses). Seules les courbes de la campagne (Melesse en vert), du centre (Griffon en rouge) et de Saint-Jacques (en noir) sont différenciées, les courbes des autres stations sont en gris. Hourly temperature variations from July 22 to 25,2019 in Rennes (GMT hours on the abscissa). Only the curves of the countryside (Melesse in green), the center (Griffon in red) and Saint-Jacques (in black) are differentiated, the curves of the other stations are in gray.

Sur l'ensemble du mois de juillet, les parcs plus étendus et en situation de léger creux topographique, comme celui des Prairies SaintMartin, affichent une différence moyenne de l'ordre de $1,2^{\circ} \mathrm{C}$ avec les stations urbaines voisines. L'îlot de fraîcheur est plus faible, de l'ordre de 0,3 à $0,4^{\circ} \mathrm{C}$, pour des parcs plus petits comme le Thabor (12 hectares) ou pour le square de Villeneuve (environ un hectare au centre sud-ouest). Ces résultats devront être confirmés par des campagnes de mesures spécifiques pour mieux apprécier la distance jusqu'à laquelle est sensible l'effet rafraîchissant des parcs en fonction de leur étendue et du type de bâti environnant.

\subsection{La question de la raréfaction du gel en ville?}

Un dernier point mérite attention, celui de la diminution de la fréquence et de l'intensité du gel en ville. Ainsi, le nombre de jours de gel sur l'ensemble de l'année 2019 a été de 26 à SaintJacques de la Lande, une valeur inférieure de 7 jours à la moyenne 1981-2010. Mais tandis que dans les stations de référence rurales de cette étude le nombre de jours de gel atteignait 32, il est resté inférieur à 10 dans les stations du centre-ville, une valeur qui correspond à la moyenne annuelle des stations littorales bretonnes comme Groix ou Belle Ile. Mieux encore, dans les trois stations de l'hyper centre servant de référence à cette étude (figure 11), seule la journée du 31 janvier 2019 a connu une température négative et de justesse $\left(-0,2\right.$ et $-0,9^{\circ} \mathrm{C}$ pour les stations urbaines centrales). A l'exception des secteurs du nord de la commune, l'ensemble de la commune intra-rocade a connu un nombre de jours de gel inférieur ou égal à 15 .

Cette situation n'est cependant pas complètement nouvelle : le graphique de la figure 12 montre ainsi que, de 2004 à 2019, le nombre de jours de gel à Saint-Jacques a fluctué entre 5 et 65 (et jusqu'à 70 jours dans les campagnes du nord de Rennes en 2010, c'est-à-dire la moyenne interannuelle de Reims, par exemple). Dans le même temps, dans le centre-ville de Rennes, ces chiffres ont varié entre 1 et 33 : 6 années sur les 16 de la série d'observation ont connu des années avec moins de 10 jours de gel. En 2014 et en 2016, déjà, seulement deux jours de gel avaient été notés dans le centre-ville: pour rappel, ces chiffres correspondent aux valeurs observées en moyenne à Nice ou sur le littoral Corse ! L'îlot de chaleur n'est donc pas seulement un phénomène qui réchauffe le climat «en moyenne » ou qui accentue l'effet des vagues de chaleur lors des nuits estivales, il s'agit d'un phénomène qui altère profondément le climat local, générant des isolats ne trouvant des équivalents qu'à plusieurs dizaines voire centaines de kilomètres de distance en fonction du paramètre 
climatique observé. Il est donc probable que le centre de Rennes connaîtra prochainement des hivers sans aucune gelée, accentuant la tendance à la baisse de la fréquence de ce phénomène déjà observée dans la région sur la figure 8 ou par Mérot et al., (2012) et dont les impacts sont déjà perceptibles sur la végétation (Mimet et al, 2009 ; Quénol et al., 2010).

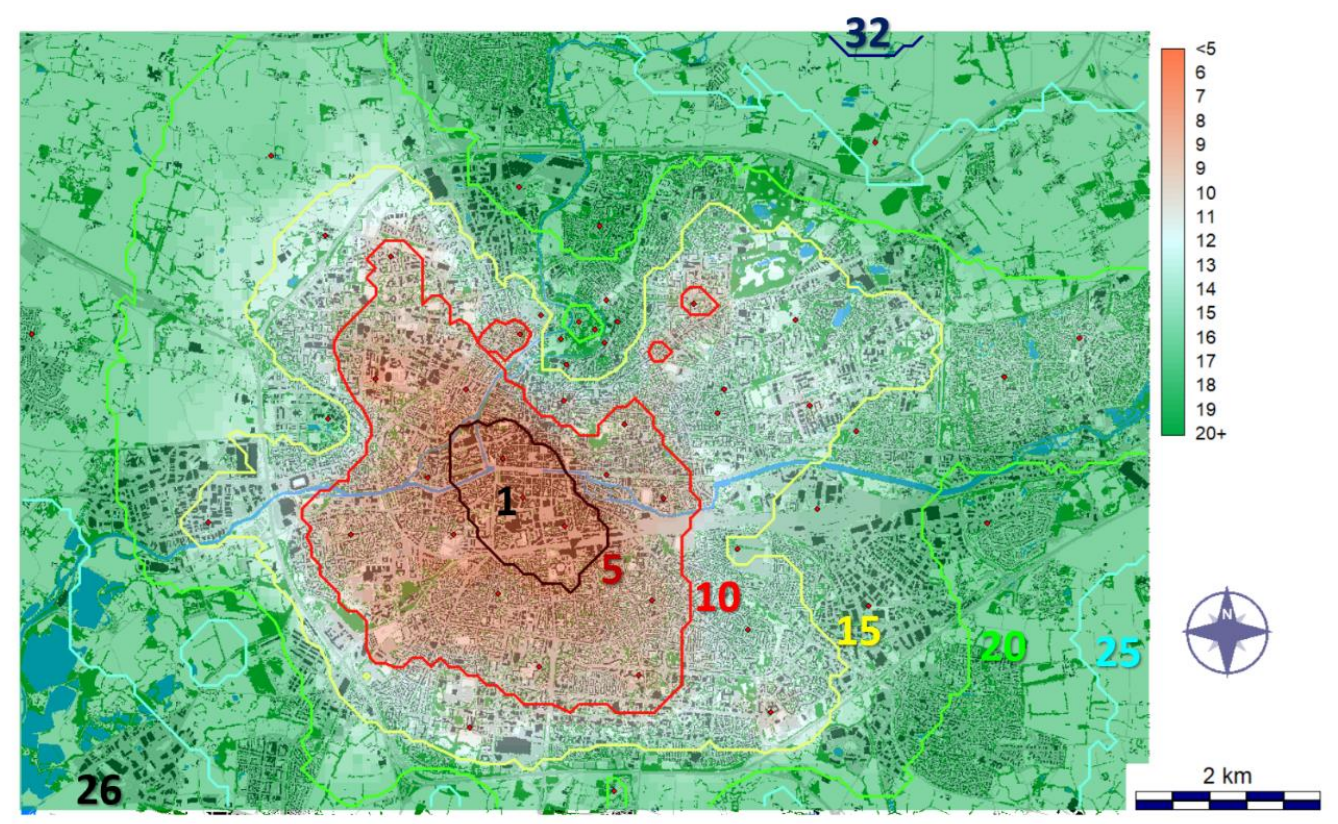

Figure 11. Nombre de jours de gel observés à Rennes en 2019. Number of frost days observed in Rennes in 2019.

Figure 12. Evolution du nombre de jours de gel pour 6 stations rennaises de 2004 à 2019. Evolution of the number of frost days for 6 stations in Rennes from 2004 to 2019.

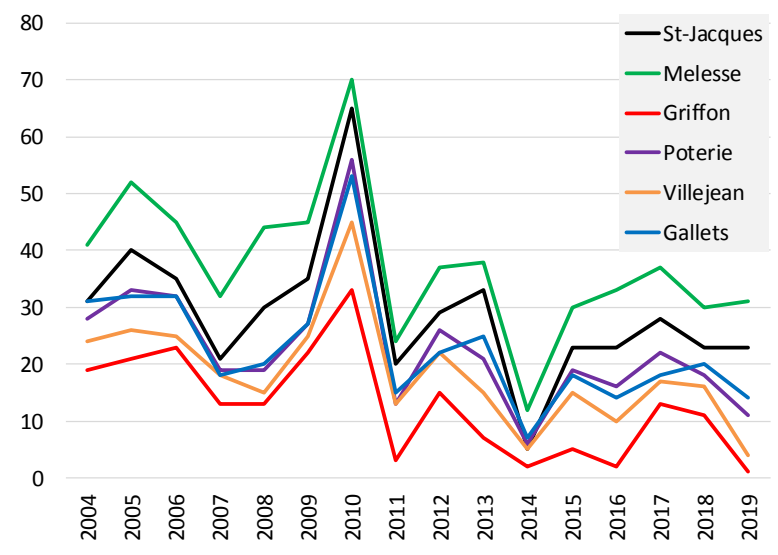

années d'observation, restant donc tributaires des particularités météorologiques des années en question. Ainsi, la dernière année de la série présentée ici, 2019, se révèle singulière avec de fortes fréquences et intensités des îlots de chaleur urbain observés aussi bien l'été (situation classique, lorsque les situations sèches et radiatives dominent) que l'hiver. A Rennes, le développement des situations d'ICU même pendant la saison froide est remarquable et tend à rendre très rare (voire quasi inexistant) le gel dans le centre-ville. Plus 
généralement, même si les seize années d'observation sont encore une période assez courte, elles semblent montrer, en plus d'une diminution sensible et continue du nombre de jours de gel en ville, une tendance à l'augmentation de l'ICU moyen et une croissance de la fréquence des ICU de forte intensité. S'il est tentant de mettre cette évolution en rapport avec l'accroissement de population $(+0,8 \%$ par an en moyenne d'après Poquet et al., 2019) et l'extension des surfaces urbanisées, il convient de rester prudent car $i$ ) l'année 2019 qui clôt cette courte série est une année assez remarquable en terme d'ICU et peut donc tendre à exagérer (voire créer artificiellement) cette tendance, et $i i$ ) on a montré que la fréquence des situations favorables à l'ICU avait aussi plutôt augmenté sur la période. La poursuite des observations devrait permettre dans les prochaines années d'avancer sur ce point.

L'autre force du réseau d'observation est la complémentarité entre les stations «historiques» complètes sur lesquelles des statistiques pluriannuelles peuvent être construites et le réseau plus léger (et, plus récemment, le suivi en temps réel que permettent les capteurs connectés) des campagnes annuelles et saisonnières : la grande densité de points de mesures (plus de 50 en 2019) permet d'obtenir des interpolations spatiales précises tenant compte de l'occupation du sol et notamment de la densité du bâti et de la végétation dont on sait qu'elles influencent fortement la configuration spatiale de l'ICU. Ainsi, à Rennes, le schéma d'ICU relativement concentrique observable dans la partie sud de l'agglomération est contesté au nord par la présence de véritables coulées vertes et topographiques qui favorisent la pénétration des influences fraîches rurales (près de $2^{\circ} \mathrm{C}$ ) jusqu'à proximité du centre-nord. Plus localement, les parcs et jardins permettent le développement d'îlots de fraîcheur de « proximité » réduisant l'ICU de quelques dixièmes de degrés en moyenne. Des campagnes de mesures spécifiques appuyées par des observations satellitaires infrarouge devront cependant venir préciser jusqu'à quel point ces îlots de fraîcheur restent perceptibles quand on s'éloigne des parcs.

La cartographie fine de l'ICU est cruciale pour identifier les secteurs à enjeu fort et aider les acteurs du territoire dans la planification urbaine. Dans la situation actuelle de poursuite de la croissance de la population et de politique simultanée de densification urbaine de la commune centre et de croissance forte des petites villes satellites (le schéma dit de la «ville archipel»; Poquet et al., 2019), la question des différentes manières de réduire l'intensité des ICU dans un contexte de changement climatique reste entière : la situation est d'autant plus complexe qu'à Rennes, $70 \%$ de la végétation présente où l'ICU est intense en ville correspond à l'espace privé (Foissard et al., 2018). Cette localisation souligne l'importance de préserver la végétation de l'espace privé et pas seulement de la végétation et des parcs publics.

Remerciements : Cette étude a été réalisée dans le cadre du programme CAPES/COFECUB Sh 941/19, CiClAMEn (Cities, Climate And vegetation: Modeling and Environmental public policies). Les recherches s'inscrivent également dans les dispositifs «Zone Atelier Armorique » et DIPEE (Dispositifs de partenariat en écologie et environnement) du CNRS. Les données de Rennes Saint-Jacques ont été acquises sur la Publithèque de Météo France : publitheque.meteo.fr. Les auteurs remercient l'ensemble des institutions et personnes (trop nombreuses pour être citées ici) qui ont participé aux campagnes de mesures depuis 2004.

\section{Références}

Alcoforado M. J., Lopes A., Alves E. D. L., Canario P., 2014. Lisbon Heat Island - Statistical Study 2004-2012. Finisterra, 98, 61-80.

Amorim M. C. C. T., Dubreuil V., Cardoso R. dos S., 2015. Modelagem espacial da ilha de calor urbana em presidente prudente (SP) - Brasil. Rev. Bras. Climatol. 16, 29-45.

Amorim M. C. C. T., Dubreuil V., 2017. Intensity of Urban Heat Islands in Tropical and Temperate Climates. Climate, (5), 91-104.

Arnfield A. J., 2003. Two decades of urban climate research: a review of turbulence, exchanges of energy and water, and the urban heat island. Int. J. Climatol. 23, 1-26.

Cantat O., 2004. L'îlot de chaleur urbain parisien selon les types de temps. Norois 191, 1-37.

Carrega P., 2013. Le climat urbain de Nice en milieu géographique contrasté : synthèse par approche inductive. Climatologie, 10, 9-34. 
Chang C.-R., Li M.-H., Chang S.-D., 2007. A preliminary study on the local cool-island intensity of Taipei city parks. Landsc. Urban Plan., 80, 386-395.

Dettwiller J., 1970. Evolution séculaire du climat de Paris. Influence de l'urbanisation. Mémorial de la Météorologie Nationale, Paris, 83.

Dubreuil V., Quénol H., Planchon O., Clergeau H., 2008. Variabilité quotidienne et saisonnière de l'îlot de chaleur urbain à Rennes : premiers résultats du programme ECORURB. XXIe Colloque de l'Association Internationale de Climatologie, Montpellier, 21, 221-227.

Dubreuil V., Quénol H., Foissard X., Planchon O., 2010. Climatologie urbaine et îlot de chaleur urbain à Rennes. In P. Clergeau (dir.) Ville et biodiversité: les enseignements d'une recherche pluridisciplinaire, Presses Universitaires de Rennes, 105-122.

Dubreuil V., Lamy C., Planchon O., 2018. Les sécheresses à Rennes : passé, présent et futur. Colloque Les risques naturels dans le contexte de changement climatique, Actes du Colloque de Cluj-Napoca, 15-21.

Escourrou G., 1991. Le climat et la ville. Nathan, Paris.

Eveno M., Planchon O., Oszwald J., Dubreuil V., Quénol H., 2016. Variabilité et changement climatique en France: analyses au moyen de la classification de Köppen et des " types de climats annuels ». Climatologie, 13, 47-70.

Fernández García F., 1996. Manual de climatologia aplicada: clima, medio ambiente y planificación. Madrid: Editorial síntesis, S.A., 285.

Foissard X., 2015. L'îlot de chaleur urbain et le changement climatique : application à l'agglomération rennaise. Thèse de doctorat de géographie de l'Université Rennes 2.

Foissard X., Dubreuil V., Quénol H., 2018. Urban heat island in mid-size city - measurements, modeling and projections for planning documents. 10th International Conference on Urban Climate-AMS, New York.

Foissard X., Dubreuil V., Quénol H., 2019. Defining scales of the land use effect to map the urban heat island in a mid-size European city: Rennes (France). Urban Climate, 29.

Gaffin S.R., Rosenzweig C., Khanbilvardi R., Parshall L., Mahani S., Glickman H., Goldberg R., Blake R., Slosberg R.B. and Hillel D., 2008. Variations in New York City's urban heat island strength over time and space. Theoretical and Applied Climatology, 94, 1-11.

Hidalgo J., Dumas G., Masson V., Petit G., Betchtel B., Bocher E., Foley M., Schoetter R., Mills, G., 2019. Comparison between local climate zones maps derived from administrative datasets and satellite observations. Urban Climate. 27, 64-89.

Hung T., Uchihama D., Ochi S. and Yasuoka Y., 2006. Assessment with satellite data of the urban heat island effects in Asian mega cities. International Journal of Applied Earth Observation and Geoinformation, 8, 34-48.

Kastendeuch P., Najjar G., Lacarrere P., Colin J., 2010.
Modélisation de l'îlot de chaleur urbain à Strasbourg. Climatologie, 7, 21-38.

Katsoulis B. D. and Theoharatos G. A., 1985. Indications of the urban heat island in Athens, Greece. Journal of Climate and Applied Meteorology, 24, 1296-1302.

Lemonsu A., Masson V., Shashua-Bar L., Erell E., Pearlmutter D., 2012. Inclusion of vegetation in the town energy balance model for modelling urban green areas. Geosci. Model Dev. 5, 1377-393.

Masson V., 2006. Urban surface modeling and the meso-scale impact of cities. Theor. Appl. Climatol., 84, 35-45.

Masson V. et al., 2019. City-descriptive input data for urban climate models: Model requirements, data sources and challenges. Urban Climate, 31, 100536.

Merot P., Dubreuil V., Delahaye., Desnos P., 2012. Changement climatique dans l'Ouest : Évaluation, impacts, perceptions. Presses Universitaires de Rennes, Coll. Espaces et Territoires.

Mestayer P.-G., Durand P., Augustin P. et al., 2005. The urban boundary-layer field campaign in marseille (ubl/cluescompte): set-up and first results. Boundary-Layer Meteorology, 114, 315-365.

Mimet A., Pellissier V., Quénol Q., Aguejdad R., Dubreuil V., Rozé F., 2009. Urbanisation induces early flowering: evidence from Platanus acerifolia and Prunus cerasus. International Journal of Biometeorology, 53, 287-298

Mohan M., Kikegawa Y., Gurjar B. R., Bhati S., Kandya A. \& Ogawa K., 2009. Assessment of urban heat island intensities over Delhi. In The seventh international conference on urban climate, Yokohama, Japan.

Morris C., Simmonds I., 2001. Quantification of the influences of wind and cloud on the nocturnal urban heat island of a large city. J. Appl. Meteor., 40(2), 169-182.

Nabucet J., 2018. Apport des données de télédétection à très haute résolution spatiale pour la cartographie de la végétation en milieu urbain. Thèse de doctorat de géographie de l'Université Rennes 2.

Oke T., 1987. Boundary Layer Climates. 2nd ed.

Oke T. R., Mills G., Christsen A., Voogt J. A., 2017. Urban Climates. Cambridge University Press.

Oliveira S., Andrade H., Vaz T., 2011. The cooling effect of green spaces as a contribution to the mitigation of 387 urban heat: A case study in Lisbon. Build. Environ., 46, 2186-2194.

ONU, 2015. World Urbanization Prospects: The 2014 Revision. United Nations, Department of Economic and Social Affairs, Population Division, ST/ESA/SER.A/366.

Pigeon G., Legain D., Durand P., Masson V., 2007. Anthropogenic heat release in an old European agglomeration (Toulouse, France). Int. J. Climatol., 27, 1969-1981.

Poquet J., Dubreuil V., Bouriau E., 2019. Vulnérabilités et atouts du bassin rennais : quelle adaptation face au changement climatique ? Notes de l'AUDIAR, 2019. 〈halshs02486493〉 
Quénol H., Dubreuil V., Mimet A, Pellissier V., Aguejdad R, Clergeau P. Bridier S., 2010. Climat urbain et impact sur la phénologie végétale printanière. La Météorologie, 68, 50-57.

Renard F., Alonso L., Fitts Y., Hadjiosif A., Comby J., 2019. Evaluation of the Effect of Urban Redevelopment on Surface Urban Heat Islands. Remote Sens., 11, 299.

Richard Y., Emery J., Dudek J., Pergaud J., Chateau-Smith C. et al., 2018. How relevant are local climate zones and urban climate zones for urban climate research? Dijon (France) as a case study. Urban Climate, 26, 258-274.
Rubel F., Kottek M., 2010. Observed and projected climate shifts 1901-2100 depicted by world maps of the KöppenGeiger climate classification. Meteorologische Zeitschrift, 19, 135-141.

Stewart I. D., Oke T. R., 2012. Local Climate Zones for Urban Temperature Studies. Bull. Am. Meteorol. Soc., 93, 1879-1900

Yang X., Li Y., Luo Z., Chan P.W., 2017. The urban cool island phenomenon in a high-rise high-density city and its mechanisms. Int. J. Climatol., 37, 890-904. 